ARTICLE

\title{
The BCL-2 pathway preserves mammalian genome integrity by eliminating recombination-defective oocytes
}

\author{
Elias Ellnati ${ }^{1,8}$, Agata P. Zielinska (10 2,8, Afshan McCarthy ${ }^{3}$, Nada Kubikova (1) ${ }^{4,5}$, Valdone Maciulyte ${ }^{1}$, \\ Shantha Mahadevaiah", Mahesh N. Sangrithi, 6,7, Obah Ojarikre, Dagan Wells (1) 4,5, Kathy K. Niakan (1) ${ }^{3}$, \\ Melina Schuh ${ }^{2} \&$ James M. A. Turner ${ }^{1 凶}$
}

DNA double-strand breaks (DSBs) are toxic to mammalian cells. However, during meiosis, more than 200 DSBs are generated deliberately, to ensure reciprocal recombination and orderly segregation of homologous chromosomes. If left unrepaired, meiotic DSBs can cause aneuploidy in gametes and compromise viability in offspring. Oocytes in which DSBs persist are therefore eliminated by the DNA-damage checkpoint. Here we show that the DNAdamage checkpoint eliminates oocytes via the pro-apoptotic BCL-2 pathway members Puma, Noxa and Bax. Deletion of these factors prevents oocyte elimination in recombination-repair mutants, even when the abundance of unresolved DSBs is high. Remarkably, surviving oocytes can extrude a polar body and be fertilised, despite chaotic chromosome segregation at the first meiotic division. Our findings raise the possibility that allelic variants of the BCL-2 pathway could influence the risk of embryonic aneuploidy.

\footnotetext{
${ }^{1}$ Sex Chromosome Biology Laboratory, The Francis Crick Institute, London NW1 1AT, UK. ${ }^{2}$ Max Planck Institute for Biophysical Chemistry, Am Fassberg 11, Göttingen 37077, Germany. ${ }^{3}$ Human Embryo and Stem Cell Laboratory, The Francis Crick Institute, London NW1 1AT, UK. ${ }^{4}$ Nuffield Department of Women's and Reproductive Health, John Radcliffe Hospital, University of Oxford, Oxford OX3 9DU, UK. ${ }^{5}$ IVI-RMA, Magdalen Centre, Oxford Science Park, Oxford OX4 4GA, UK. ${ }^{6}$ Duke-NUS Graduate Medical School, Singapore 119077, Singapore. ${ }^{7}$ Department of Reproductive Medicine, KK Women's and Children's Hospital, Singapore 229899, Singapore. ${ }^{8}$ These authors contributed equally: Elias Ellnati and Agata P. Zielinska. ${ }^{凶}$ email: james.turner@crick.ac.uk
} 
$\Lambda$ pate 1 defining feature of sexual reproduction is meiosis, when paternal and maternal homologous chromosomes synapse and recombine. In mammals, both processes are dependent on meiotic DNA double-strand breaks (DSBs) generated by the SPO11 enzyme ${ }^{1-3}$. DSBs are subsequently processed by the recombination machinery, which includes the RecA homolog $\mathrm{DMC1}^{4,5}$ and mismatch repair protein $\mathrm{MSH}^{6,7}$, to form crossovers or non-crossovers ${ }^{8}$. Crossovers allow the homologous pair to be recognised as a single entity by spindle microtubules, and hence facilitate accurate chromosome segregation. Defects in meiotic chromosome segregation produce aneuploid gametes and are the leading cause of congenital disorders and pregnancy loss ${ }^{9,10}$. Chromosome segregation errors arise more frequently and are more likely to remain undetected in the female than the male germline $e^{9,10}$. Understanding the pathways that eliminate defective oocytes could permit the development of therapies that enhance fertility in women, by improving the quality of the oocyte pool present at ovulation ${ }^{11,12}$.

Historically, oocyte elimination in mice has been attributed to three quality control mechanisms. Meiotic silencing, the inactivation of genes on asynapsed chromosomes, triggers oocyte loss when one or two chromosomes are asynapsed ${ }^{13}$. A second mechanism, the synapsis checkpoint, triggers oocyte loss when asynapsis is more extensive, and operates even when programmed DSBs are not formed, e.g., in Spo11 $1^{-1-}$ females ${ }^{14-16}$. HORMAD1 and HORMAD2 are implicated in these first two quality control pathways ${ }^{15,16}$. The third mechanism, the DNAdamage checkpoint, eliminates oocytes with persistent DNA damage. Oocytes with defective recombination repair, e.g., $D m c 1^{-1-4,5}$ and $M s h 5^{-1-6,7}$, exhibit both persistent DNAdamage and chromosome asynapsis, and thus may be eliminated by the combined effects of the synapsis and DNA-damage checkpoint. Several components of the DNA-damage checkpoint have now been identified: repair of lingering DSBs is impeded by the SUMO ligase RNF212, leading to the activation of the checkpoint protein $\mathrm{CHK} 2$ and thereafter $\mathrm{p} 53$ and $\mathrm{p} 63^{17,18}$.

Interestingly, recent data has shown that $S p o 11^{-/-}$females exhibit spontaneous DNA damage ${ }^{19}$ despite no programmed meiotic DSBs being formed ${ }^{2,3}$. Elimination of Spo11 $1^{-1-}$ oocytes also requires RNF212 and CHK2 ${ }^{18}$. Furthermore, the ablation of HORMAD2 reduces the abundance of DNA-damage markers in Spo11 ${ }^{-1-}$ oocytes $^{18}$. These findings call into question the existence of the synapsis checkpoint, and suggest that the DNAdamage checkpoint may eliminate oocytes in mutants lacking programmed DSB-formation. A prediction of this hypothesis is that the deletion of other checkpoint effectors should rescue oocyte elimination both in mutants with persistent meiotic DSBs, e.g., $\mathrm{Dmcl}^{-/-}$and $\mathrm{Msh}^{-/-}$females, and those lacking programmed DSBs, e.g., Spo11 ${ }^{-1-}$ females.

To date, the checkpoint factors acting downstream of CHK2/ p53/p63 to trigger oocyte apoptosis have not been identified. The p53 and p63 transcription factors bind many genomic sites and regulate multiple cellular responses ${ }^{20}$. Among their mitotic targets are intrinsic apoptosis pathway components PUMA, NOXA, and $\mathrm{BAX}^{20}$. PUMA and NOXA inactivate members of the prosurvival BCL-2 family, which in turn relieves inhibition of proapoptotic factors BAX and BAK and promotes cell death ${ }^{21,22}$. PUMA may also directly bind and activate BAX/BAK ${ }^{23}$. PUMA and NOXA mediate oocyte loss in response to postnatal, $\gamma$ irradiation (IR)-induced $\mathrm{DSBs}^{24}$, but whether they do so in response to lingering SPO11-catalysed DSBs is not known. Establishing the extent of similarity between exogenous and meiotically-programmed DNA damage responses is clinically important, because it will reveal whether therapies that protect germ cells from genotoxic agents could be useful for the treatment of meiotic infertility. BAX is required for oocyte elimination during development ${ }^{25-27}$ and in response to chemotherapy ${ }^{28}$. However, its role in oocyte loss following IR-induced DSBs or persistent meiotic DNA damage has not been defined.

Here we demonstrate that PUMA, NOXA, and BAX are components of the DNA-damage checkpoint that eliminates $\mathrm{Dmcl}^{-1-}$ and $\mathrm{Msh}^{-1-}$ oocytes. The deletion of BCL-2 components does not rescue oocyte loss in Spo11 ${ }^{-1-}$ females, demonstrating that the oocyte quality controls operating in these models are genetically dissociable. We also determine the effects of disabling the DNA-damage on oocyte chromosome segregation, maturation, and embryo development.

\section{Results}

Puma and Noxa mediate oocyte loss in Dmc1 and Msh5 nulls. Exposure to $0.45 \mathrm{~Gy}$ of IR at postnatal day 5 (P5) results in the elimination of all primordial follicles by P10 and subsequent infertility in wild-type female mice ${ }^{29,30}$. The same effect is observed in $\mathrm{Noxa}^{-1-}$ females ${ }^{24}$. However, in $\mathrm{Puma}^{-1-}$ and Puma $^{-1-}$ Noxa $^{-1-}$ females, $16 \%$ and $52 \%$ of oocytes are protected from IR-induced elimination, respectively, relative to nonirradiated mice of the same genotypes ${ }^{24}$. Irradiated Puma ${ }^{-1-}$ $\mathrm{Noxa}^{-1-}$ females are fertile ${ }^{24}$, demonstrating that some oocytes survive beyond P10. In line with these published observations, we found that while primordial follicles were absent in irradiated Puma $^{+/-} \mathrm{Noxa}^{+/-}$controls at P21, 30\% of primordial follicles survived elimination in irradiated $\mathrm{Puma}^{-1-} \mathrm{Noxa}^{-1-}$ females (Supplementary Fig. 1a). These findings support the involvement of Puma and Noxa in IR-induced oocyte loss 24 .

In $\mathrm{Dmcl}^{-1-}$ and $M s h 5^{-1-}$ females, meiotic recombination is severely impaired resulting in persistent DNA-damage and chromosome asynapsis. DMC1 acts early in recombination, by coating resected DSBs to facilitate single-strand invasion and recombination repair ${ }^{31,32}$, while MSH5 acts later to stabilise double-Holliday junctions ${ }^{33,34}$. In $D m c 1^{-1-}$ and $M s h 5^{-1-}$ females, persistent DSBs cause oocyte loss that is already evident at P0 (birth) ${ }^{4-7}$, and by P21 primordial and more advanced follicles are scarce (Fig. 1a). We examined whether P21 oocyte counts in $D m \mathrm{ml}^{-1-}$ and $M s h 5^{-1-}$ females were influenced by the deletion of Puma and Noxa. Ovaries from $\mathrm{Dmcl}^{-1-}$ and $M s h 5^{-I-}$ females lacking either Puma only, or Noxa only, contained no primordial follicles. A few more advanced follicles were observed in these models, but not at an abundance different to that in $D m c 1^{-I-}$ and $M s h 5^{-I-}$ females at a statistical significance of $p<0.05$ (Supplementary Fig. 1b, c). However, Dmc1 ${ }^{-1-}$ Puma $^{-1-}$ Noxa $^{-1-}$ and $\mathrm{Msh}^{-1-} \mathrm{Puma}^{-1-} \mathrm{Noxa}^{-1-}$ P21 ovaries contained primordial as well as more developmentally advanced follicles (Fig. 1b). Total oocyte counts in $D m c 1^{-/-}$ Puma $^{-1-}$ Noxa $^{-1-}$ and Msh5 $^{-1-}$ Puma $^{-1-}$ Noxa $^{-1-}$ females were $38 \%$ and $34 \%$ of those observed in $\mathrm{Puma}^{-1-} \mathrm{Noxa}^{-1-}$ females, respectively (Fig. 1c). Combined immunostaining for the chromosome axis protein SYCP3 and the DSB marker RPA2 confirmed that at P0 meiotic DNA damage was present in $\mathrm{Dmcl}^{-1-} \mathrm{Puma}^{-1-} \mathrm{Noxa}^{-1-}$ and $\mathrm{Msh}^{-1-} \mathrm{Puma}^{-1-} \mathrm{Noxa}^{-1-}$ oocytes (Supplementary Fig. 2a). At P0 the mean RPA2 count was similar between $\mathrm{Dmcl}^{-1-} \mathrm{Puma}^{-1-} \mathrm{Noxa}^{-1-}$ and $\mathrm{Dmcl}^{-1-}$, and between $\mathrm{Msh}^{-1-} \mathrm{Puma}^{-1-} \mathrm{Noxa}^{-1-}$ and $\mathrm{Msh}^{-1-}$ oocytes, at this age (Supplementary Fig. 2b). Oocyte survival is therefore due to checkpoint attenuation, rather than activation of an alternative DSB-repair pathway. We conclude that Puma and Noxa cooperate in the downstream output of the oocyte DNA-damage checkpoint.

Bax deletion rescues oocyte loss in Dmc1 and Msh5 nulls. We tested whether Bax drives oocyte elimination in response to IR or persistent meiotic DNA damage. Bax deletion in non-irradiated 
a
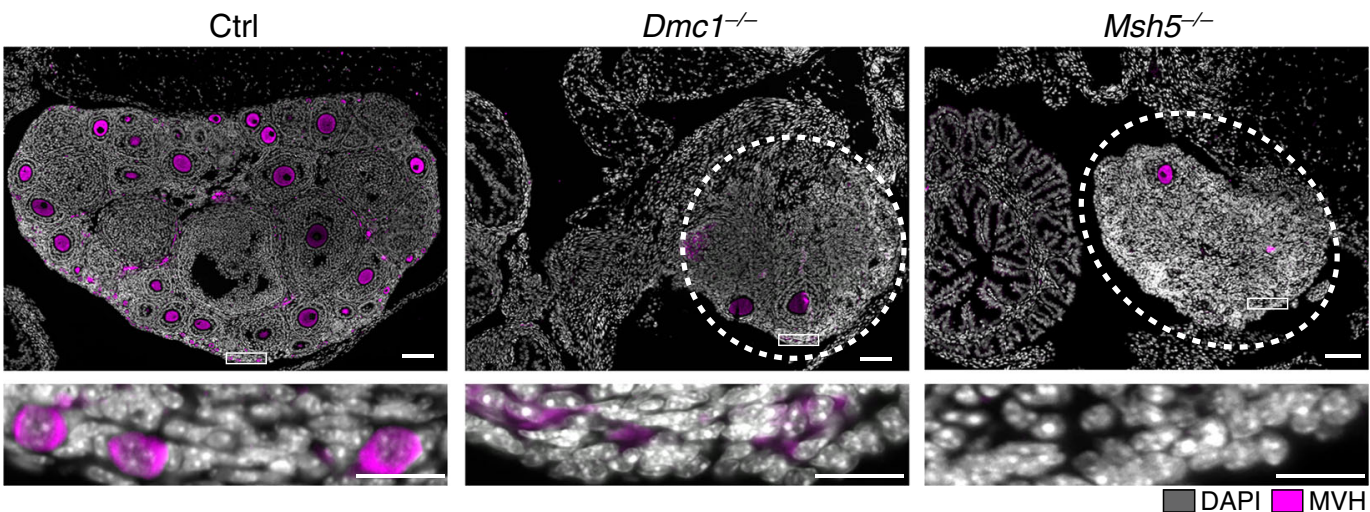

b
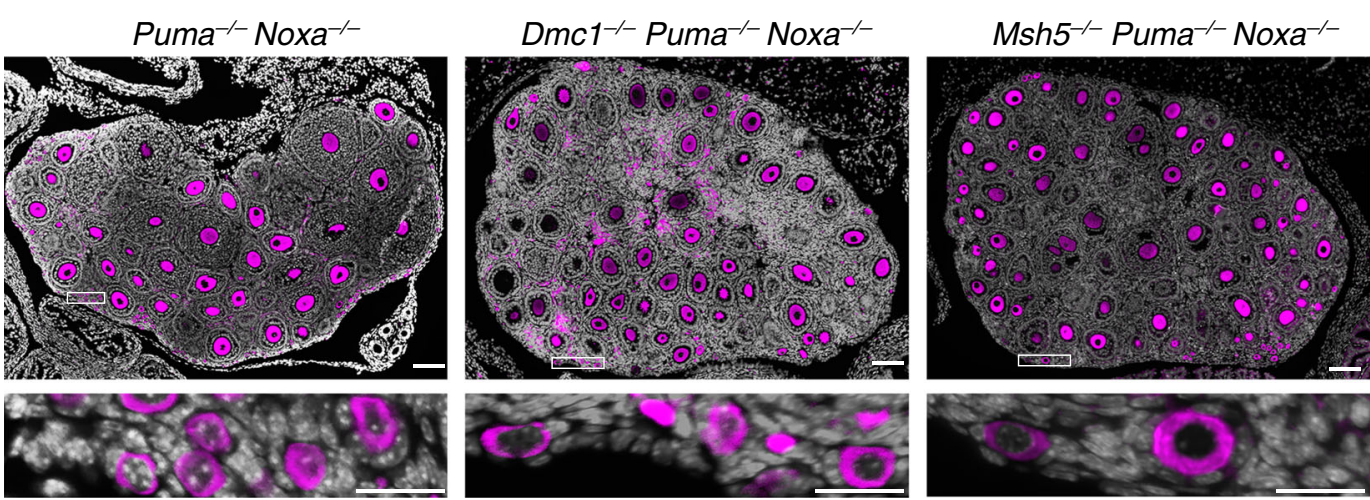

C

Primordial $\square$ Growing
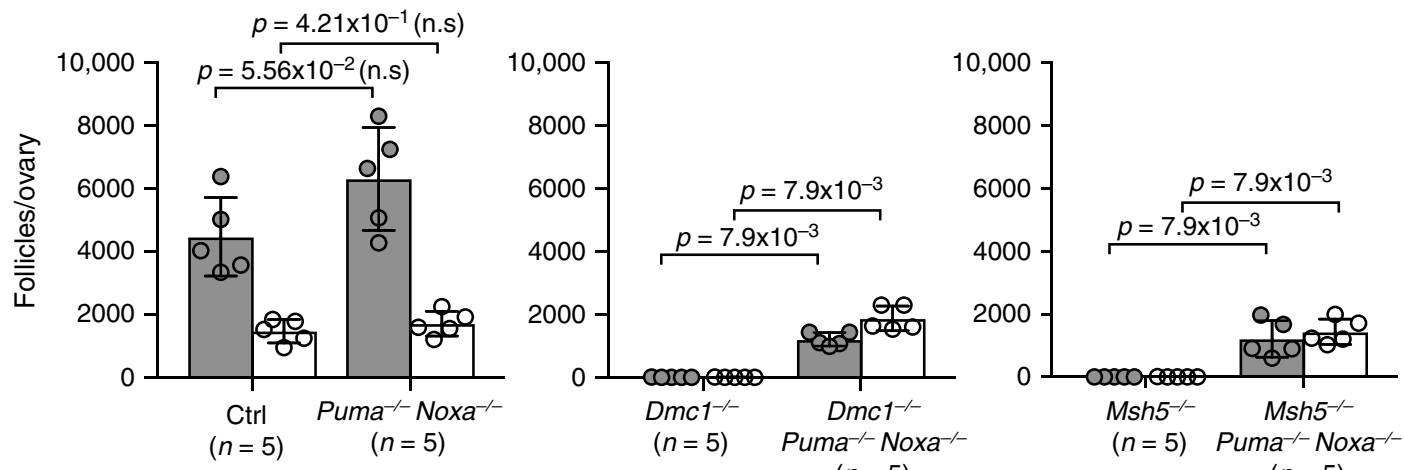

$(n=5)$

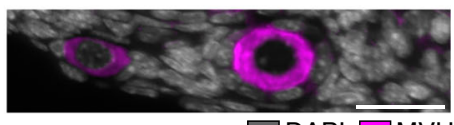

$\square$ DAPI $\square \mathrm{MVH}$

Fig. 1 Puma and Noxa deletion rescues oocyte loss in Dmc1 and Msh5 nulls. a, b P21 ovary sections immunostained for oocyte marker MVH (magenta). Control (Ctrl) is Dmc1+/- Puma ${ }^{+/-} \mathrm{Noxa}^{+/-}$. White rectangles in upper panels show cortex, which is magnified in lower panels. Dotted circles outline degenerated ovaries ( $n=5$ females, $N=3$ experimental repetitions). Scale bar in upper panels $100 \mu \mathrm{m}$, scale bars in micrographs $25 \mu \mathrm{m}$. c Primordial and total follicle quantitation at P21. Data are represented as mean \pm standard deviation. Error bars indicate $95 \%$ confidence intervals. Two-sided Mann-Whitney test was used to calculate $p$-values.

females resulted in a slightly higher number of primordial follicles compared to their wild-type counterparts, although the difference was not statistically significant at $p<0.05$. However, Bax deletion fully rescued IR-induced oocyte loss (Fig. 2a, b). Primordial follicle counts in irradiated $\mathrm{Bax}^{-1-}$ females were equivalent to those in non-irradiated $\mathrm{Bax}^{-1-}$ mice (Fig. 2b). In addition, while irradiated control females were sterile, irradiated $\mathrm{Bax}^{-1-}$ females were fertile. The mean litter size from irradiated $\mathrm{Bax}^{-1-}$ mothers was slightly lower but not significantly different $($ at $p<0.05)$ to that from non-irradiated $\mathrm{Bax}^{-/-}$and $\mathrm{Bax}{ }^{+/-}$mothers (Fig. 2c).

The deletion of Bax also enabled oocyte survival in $\mathrm{Dmcl}^{-1-}$ and $M s h 5^{-I-}$ females (Fig. 2d). At P21, primordial follicles were present, and the total oocyte count in $\mathrm{Dmcl}^{-1-} \mathrm{Bax}^{-1-}$ and
Msh5 $5^{-I-} \mathrm{Bax}^{-/-}$females was $31 \%$ and $26 \%$ of that in $\mathrm{Bax}^{-/-}$ females, respectively (Fig. 2e). We conclude that like PUMA and NOXA, BAX contributes to oocyte loss in response to both IR and persistent meiotic DNA damage. In addition, our finding that Bax deletion fully rescues IR- but not meiotic DSB-induced oocyte elimination demonstrates that the effector responses to these two sources of DNA damage are not identical.

DSB markers eventually diminish in rescued oocytes. We established whether meiotic DSBs are eventually repaired in rescued oocytes. To do this, we examined whether RPA2 foci persisted in oocytes later in development, at P7. For this analysis 
a

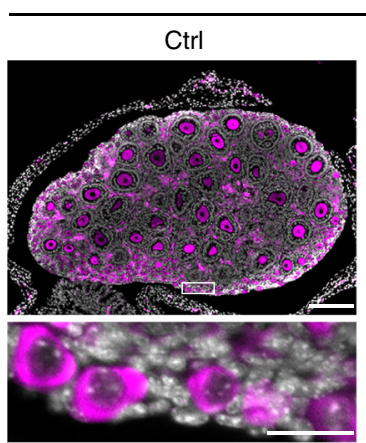

Non-irradiated

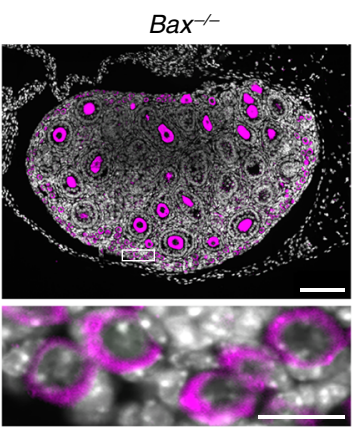

\section{Irradiated}

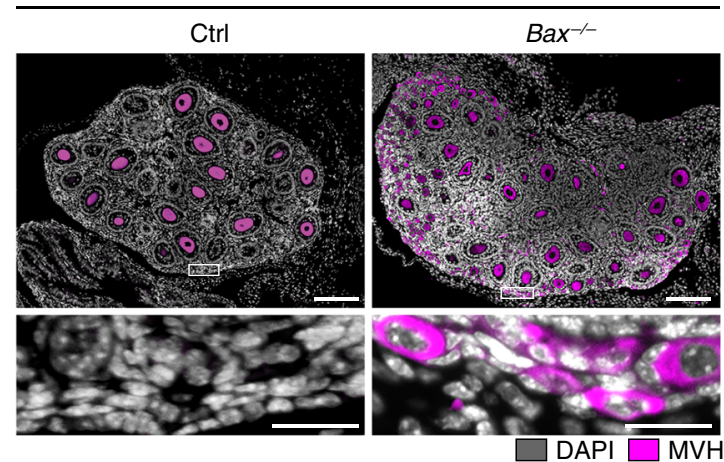

b

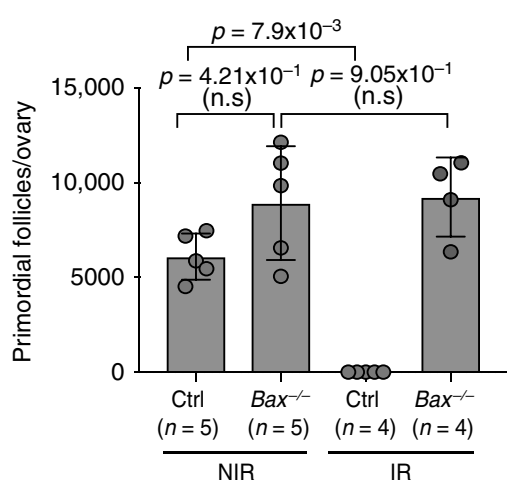

C

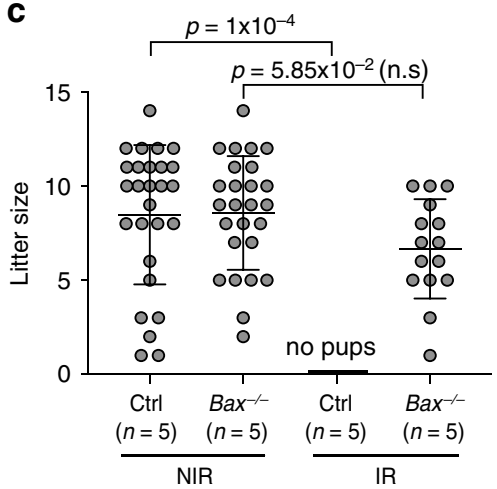

d
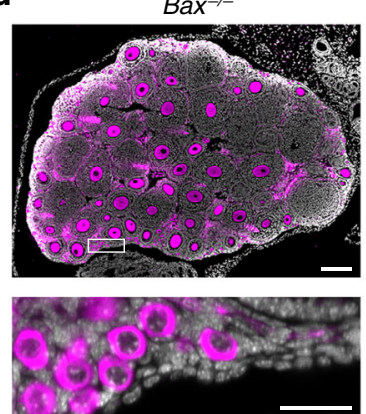

$\mathrm{Dmc1}^{-/-} \mathrm{Bax}^{-/}$
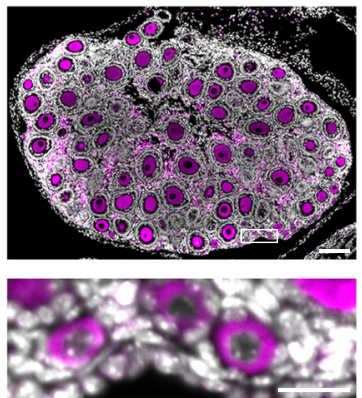

$\mathrm{Msh}^{-/-} \mathrm{Bax}^{-/-}$
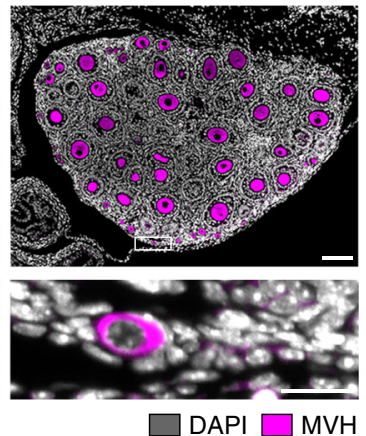

e

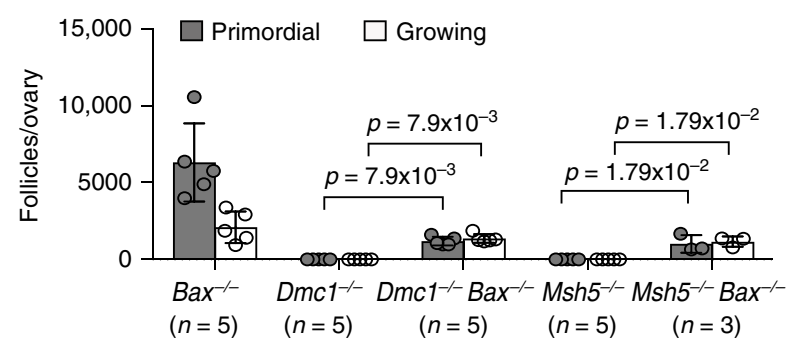

Fig. 2 Bax deletion rescues oocyte loss in Dmc1 and Msh5 nulls. a P10 ovary sections immunostained for oocyte marker MVH (magenta), harvested following $0.45 \mathrm{~Gy}$ IR-exposure at P5 (versus non-irradiated; NIR). Control (Ctrl) is Bax ${ }^{+/-}$. White rectangles in upper panels show cortex, which is magnified in lower panels. b Primordial follicle quantitation at P10. Data are represented as mean \pm standard deviation. Error bars indicate $95 \%$ confidence intervals. Two-sided Mann-Whitney test was used to calculate $p$-values. $\mathbf{c}$ Litter sizes from 6-week-old NIR or IR-treated females (five mating pairs for each group). Each circle represents a litter. Data are represented as mean \pm standard deviation. Error bars indicate $95 \%$ confidence intervals. Two-sided Mann-Whitney test was used to calculate $p$-values. d P21 ovary sections immunostained for oocyte marker MVH (magenta). e Primordial and total follicle quantitation at P21. Note that $D \mathrm{mc}^{-1-}$ and $\mathrm{Msh}^{-/-}$data is the same as that used in Fig. 1. In all panels, scale bar $100 \mu \mathrm{m}$, scale bars in micrographs $25 \mu \mathrm{m}$. Data are represented as mean \pm standard deviation. Error bars indicate $95 \%$ confidence intervals. Two-sided Mann-Whitney test was used to calculate $p$-values. 
we focused on the $\mathrm{Dmcl}^{-1-} \mathrm{Puma}^{-1-} \mathrm{Noxa}^{-1-}$ model, in which RPA2 foci were particularly abundant at P0 (Supplementary Fig. 2a, b). Since axis proteins including SYCP3 disappear postnatally, we identified oocytes using the germ cell-specific marker, GCNA (Supplementary Fig. 2a). The mean RPA2 count at this age was 10 -fold lower than that at P0 (Supplementary Fig. 2b). Since oocyte rescue in $D m c 1^{-1-} \mathrm{Puma}^{-1-} \mathrm{Noxa}^{-1-}$ females was incomplete, with one-third of the oocyte pool escaping elimination, this reduction may have reflected preferential survival of oocytes with fewer DSBs. However, this hypothesis could not fully explain the RPA2 decrease: all Dmc1-I- $\mathrm{Puma}^{-1-} \mathrm{Noxa}^{-1-}$ oocytes contained more than $100 \mathrm{RPA} 2$ foci at P0, while more than one half exhibited an RPA2 count of zero at P7 (Supplementary Fig. 2b). We also observed $\mathrm{Dmcl}^{-1-} \mathrm{Puma}^{-1-}$ Noxa ${ }^{-1-}$ oocytes at P7 containing few or no foci for a second DSB marker, RAD51 (Supplementary Fig. 2d, e). We conclude that in a cohort of surviving Dmc1 $1^{-1-}$ Puma ${ }^{-1-}$ Noxa $^{-1-}$ oocytes DSB counts diminish during maturation, possibly reflecting DSB repair.

Dmc1 null oocytes exhibit aberrant chromosome segregation. Our data showed that the co-deletion of Puma and Noxa, or deletion of Bax only, permitted survival of recombinationdefective oocytes to P21. However, it was unclear whether the rescued oocytes would mature into eggs that could be fertilised. To achieve competence for fertilisation, oocytes must resume meiosis and undergo a cascade of events. These include the interaction of chromosomes with spindle microtubules, stable alignment of chromosome pairs at the spindle equator, and subsequent partitioning of homologous chromosomes, so that one member of each pair remains in the egg while the other is segregated to the polar body. We assessed each of these events, focusing on the Dmc1-1- Puma ${ }^{-1-}$ Noxa $^{-1-}$ model.

Germinal vesicle-stage oocytes persisted in $\mathrm{Dmcl}^{-1-} \mathrm{Puma}^{-1-}$ $\mathrm{Noxa}^{-1-}$ females at 8-10 weeks, albeit at reduced numbers relative to control $\mathrm{Dmcl}^{+/-} \mathrm{Puma}^{+/-} \mathrm{Noxa}^{+/-}$females (Supplementary Fig. 3a), as expected given findings at P21 (Fig. 1c). However, in contrast to the control oocytes, which are surrounded by easily dissociable cumulus cells ${ }^{35}$ (Supplementary Fig. 3b; top panel), germinal vesicle-stage oocytes from $\mathrm{Dmcl}^{-/-}$ Puma $^{-1-}$ Noxa $^{-1-}$ females were usually surrounded by several layers of tightly-associated cumulus cells resistant to dissociation by mechanical pipetting, indicative of incomplete growth (Supplementary Fig. 3b; bottom panel). Such non-easily dissociable cumulus oocyte complexes are similar to compact cumulus cells that surround immature GV oocytes from prepubertal mice ${ }^{36,37}$. Thus, if oocytes from $\mathrm{Dmcl}^{-1-} \mathrm{Puma}^{-1-} \mathrm{Noxa}^{-1-}$ females could complete meiosis and become successfully fertilised, these events would have to rely on the characteristic ovarian follicle pool persisting in the absence of DMC1, PUMA, and NOXA.

To fully assess the developmental potential of these $D m c 1^{-1-}$ Puma $^{-1-} \mathrm{Noxa}^{-1-}$ germinal vesicle-stage oocytes, we fluorescently labelled their chromosomes (H2B-mRFP) and microtubules (MAP4-mEGFP) and followed homologue behaviour in real-time. Morphological analysis of oocytes after 19-21 h in culture showed that polar body extrusion occurred in the majority of oocytes from control females. In contrast, polar body extrusion took place in only $41 \%$ of $\mathrm{Dmcl}^{-1-} \mathrm{Puma}^{-1-} \mathrm{Noxa}^{-1-}$ oocytes (Supplementary Fig. 3c), and was delayed by a mean of 7 $\mathrm{h}$, suggesting a defect in chromosome segregation. Consistent with this finding, live-cell imaging of $\mathrm{Dmcl}^{-/-} \mathrm{Puma}^{-1-}$ Noxa-l- oocytes showed severe chromosome congression defects. The equatorial metaphase configuration typical of controls was never achieved, and instead homologous chromosomes were scattered along the spindle length (Fig. 3a and
Supplementary Movie 1). As meiosis progressed, Dmc1-/Puma $^{-1-} \mathrm{Noxa}^{-1-}$ oocytes exhibited an elevation in spindle length and volume relative to controls (Supplementary Fig. 3d, e). Of those oocytes that extruded a polar body, $71 \%$ exhibited tripolar anaphase (Fig. 3a and Supplementary Movie 1). Kinetochore immunostaining at metaphase I revealed biorientated chromosome bivalents in control and in $\mathrm{Dmcl}^{+/+} \mathrm{Puma}^{-1-}$ Noxa ${ }^{-1-}$ females. However, in $\mathrm{Dmcl}^{-1-} \mathrm{Puma}^{-1-} \mathrm{Noxa}^{-1-}$ females, all chromosomes were present as univalents and hence crossovers were never observed (Fig. 3b; see legend for quantitation). Thus, while DMC1 may not be required for repair of meiotic DSBs in maturing oocytes (Supplementary Fig. 2a, b), it is essential to ensure that such repair produces crossovers. Crossover defects are a well-established cause of chromosome mis-segregation in oocytes ${ }^{38}$ and therefore likely explain the segregation defects we observe in $\mathrm{Dmcl}^{-1-} \mathrm{Puma}^{-1-} \mathrm{Noxa}^{-1-}$ females.

We assayed chromosome behaviour in $\mathrm{Dmcl}^{-1-} \mathrm{Puma}^{-1-}$ Noxa $a^{-1-}$ oocytes that had achieved polar body extrusion. Triple mutant metaphase II oocytes assembled meiotic spindles, and the majority of chromosomes achieved alignment at the metaphase plate (Fig. 3c, right panel). This finding suggests that the chromosome congression defect observed at metaphase I is likely a consequence of unpaired univalents, rather than reflecting an intrinsic inability of $\mathrm{Dmcl}^{-1-} \mathrm{Puma}^{-1-} \mathrm{Noxa}^{-1-}$ oocytes to achieve chromosome alignment. All the metaphase II oocytes were chromosomally abnormal, with losses or gains of whole chromosomes, as well as the presence of single chromatids resulting from premature sister separation at anaphase I (Fig. 3d, Supplementary Fig. 3f, left panels, and Supplementary Table 1). These defects are a consequence of Dmcl loss, as most control and $\mathrm{Dmcl}^{+/+} \mathrm{Puma}^{-1-} \mathrm{Noxa}^{-1-}$ oocytes cultured under these conditions were euploid (Fig. 3d, Supplementary Fig. 3f, right panels, and Supplementary Table 1). We conclude that in the absence of Puma and Noxa, recombination-defective oocytes not only escape apoptosis, but also attempt to segregate their chromosomes, generating MII oocytes with aberrant chromosome complements.

Dmc1 null oocytes can support fertilisation. Two defining events of fertilisation are extrusion of a second polar body and formation of a distinct male and female pronucleus within the resulting zygote. We assessed using live imaging whether these events took place following natural (non-superovulated) matings between $\mathrm{Dmcl}^{-I-} \mathrm{Puma}^{-1-} \mathrm{Noxa}^{-I-}$ mothers and wild type fathers. The mean number of zygotes derived from $D m c 1^{-1-}$ Puma $^{-1-}$ Noxa $^{-1-}$ mothers was not significantly different at $p<$ 0.05 to that derived from control mothers (Supplementary Fig. 4a). We obtained similar results from crosses between Dmc1 $1^{-1-} \mathrm{Bax}^{-1-}$ mothers and wild type fathers (Supplementary Fig. 4b). Thus, Dmc1 ${ }^{-1-} \mathrm{Puma}^{-1-} \mathrm{Noxa}^{-1-}$ and $\mathrm{Dmcl}^{-1-}$ $\mathrm{Bax}^{-1-}$ eggs can support fertilisation.

We used low-coverage whole-genome sequencing (less than $0.01 \times$ coverage) to examine the chromosome complement of zygotes derived using Dmc1-I- $\mathrm{Puma}^{-1-} \mathrm{Noxa}^{-1-}$ eggs and their associated polar bodies. As predicted from the striking meiotic mis-segregation phenotypes, all $\mathrm{Dmcl}^{-1-} \mathrm{Puma}^{-1-}$ Noxa ${ }^{-1-}$ zygotes displayed complex aneuploidy, harbouring gains and losses affecting three or more chromosomes (Fig. 4a and Supplementary Fig. 4c). All of their associated polar bodies were also abnormal, confirming an oocyte meiotic defect with a catastrophic impact on chromosome segregation. When comparing aneuploidies detected in zygotes and corresponding polar bodies, a high degree of reciprocity was observed. For example, most chromosomal losses in polar bodies derived from $D m c 1^{-/-}$ 
a

Meiosis in live oocytes

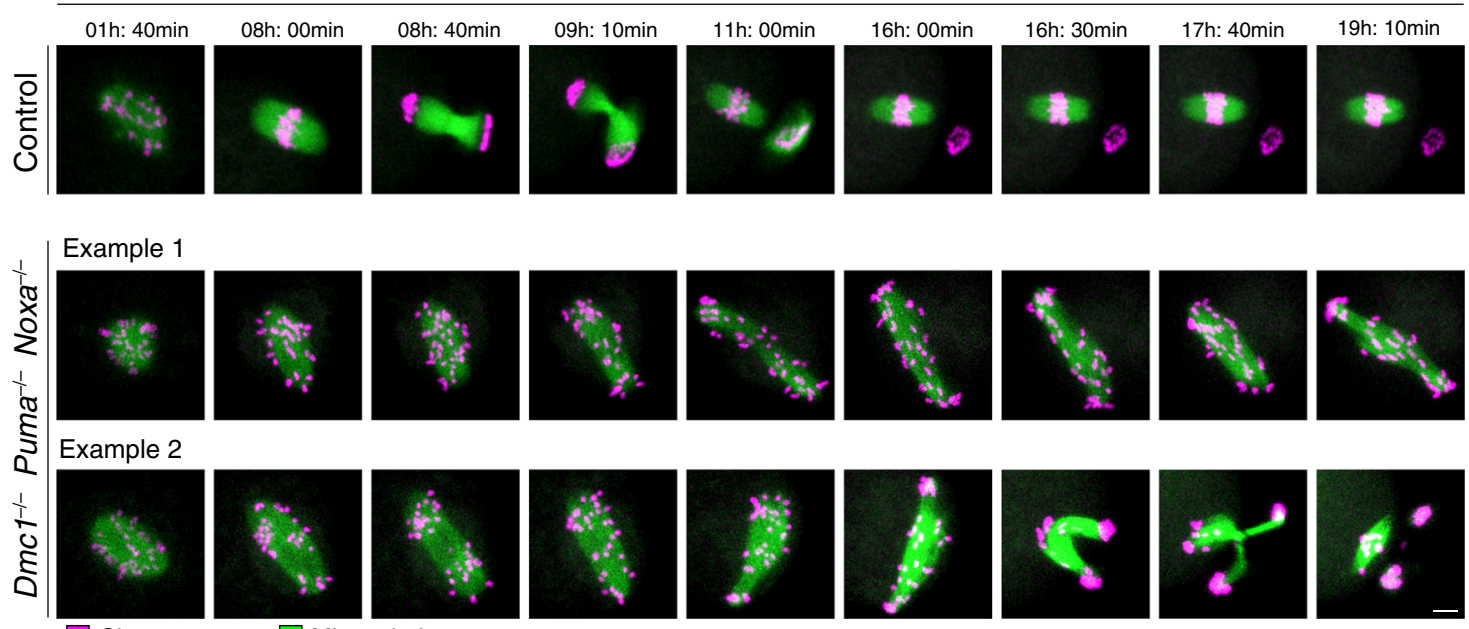

$\square$ Chromosomes $\square$ Microtubules

b

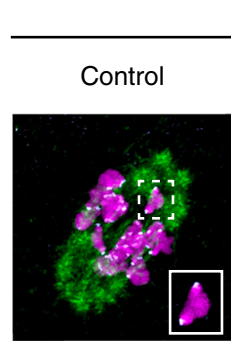

$\square$ Chromosomes

d

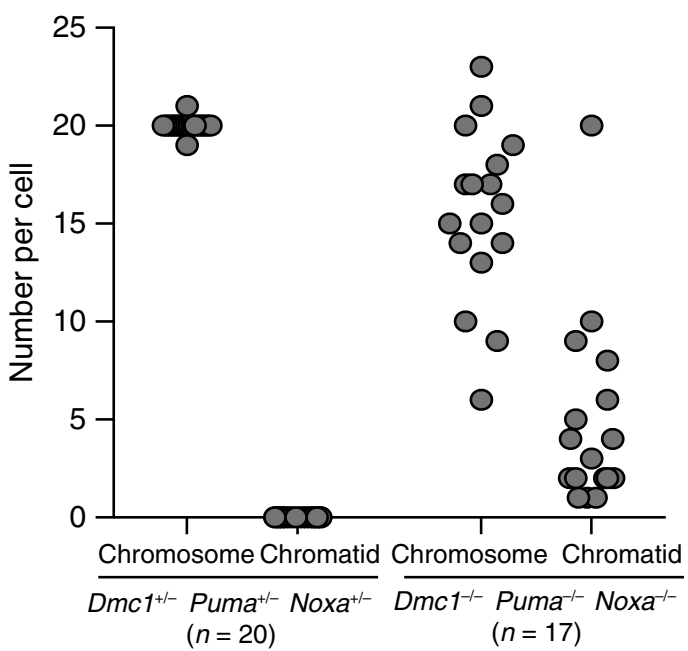

C

Metaphase II

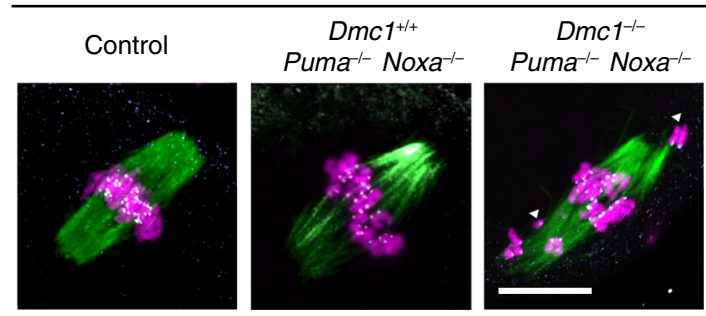

$\square$ Chromosomes $\square$ Microtubules $\square$ Kinetochores

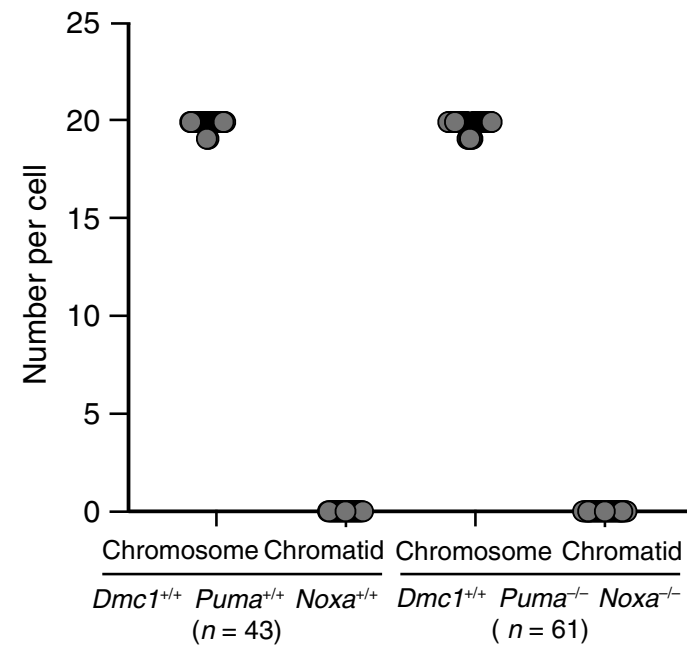

Fig. 3 Dmc1 $^{-1-}$ Puma $^{-/}$Noxa $^{-/-}$oocytes resume meiosis. a Different stages of meiosis determined from live imaging in control $(N=3$ experimental repetitions; $n=7$ females; $n=39$ oocytes) and $\mathrm{Dmc}^{-/-}$Puma $^{-/-}$Noxa ${ }^{-/-}$oocytes ( $N=3$ experimental repetitions; $n=7$ females; $n=60$ oocytes) expressing green fluorescent protein-microtubule-associated protein 4 (mEGFP-MAP4; green), and histone 2B-monomeric red fluorescent protein 1 (H2B-mRFP1; magenta). Images show z-projections of 21 sections, every $3 \mu \mathrm{m}$. Scale bar, $10 \mu \mathrm{m}$. Time displayed in hours:minutes. b, c Representative images of chromosomes (Hoechst, magenta), kinetochores (CREST, white), and microtubules ( $\alpha$-tubulin, green) in metaphase I and metaphase II

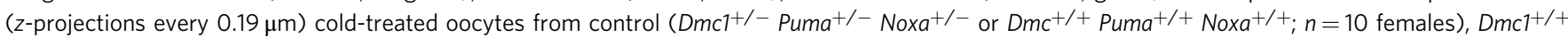
Puma $^{-/-}$Noxa $^{-/-}(n=8$ females $)$ and Dmc1 ${ }^{-/-}$Puma ${ }^{-/-}$Noxa ${ }^{-/-}(n=2$ females $)$. Insets show a magnification of a meiosis I bivalent chromosome (left and middle) or two prematurely dissociated univalent chromosomes (right). All oocytes preserved at metaphase I had all 20 chromosomes in either the bivalent or in the univalent morphology. Scale bar, $10 \mu \mathrm{m}$. d Graph representing the number of chromosomes and chromatids per metaphase II oocyte (left graph: $N=2$ experimental repetitions; $n=2$ control Dmc1+/-Puma $/ /-$ Noxa $a^{+/-}$females, $n=20$ oocytes; $n=2$ Dmc $^{-/-}$Puma ${ }^{-/-}$Noxa $^{-/-}$females, $n=17$ oocytes; right graph: $N=2$ experimental repetitions; $n=8$ control Dmc $+/+$ Puma ${ }^{+/+}$Noxa $/ /+$females, $n=43$ oocytes; $n=8$ Dmc $^{+/+}$Puma ${ }^{-/-}$ Noxa ${ }^{-1}$ females, $n=61$ oocytes). 
a

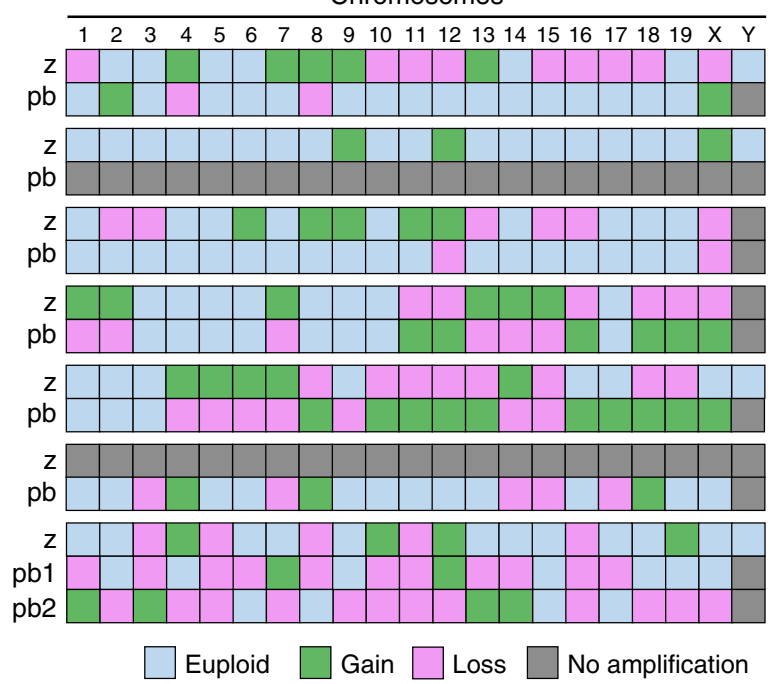

b

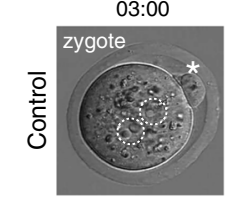

22:00
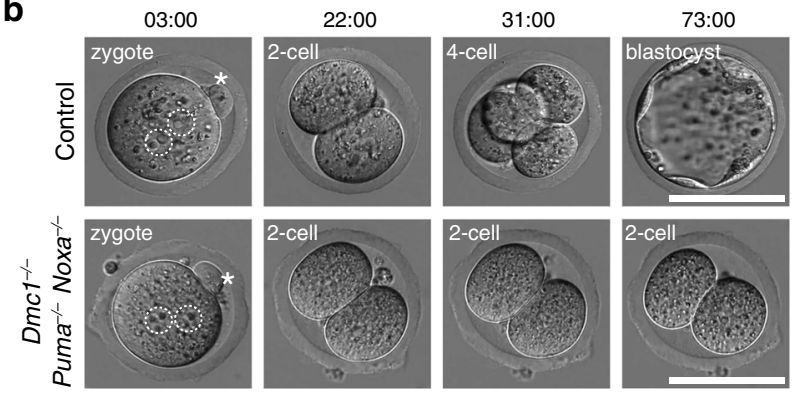

C

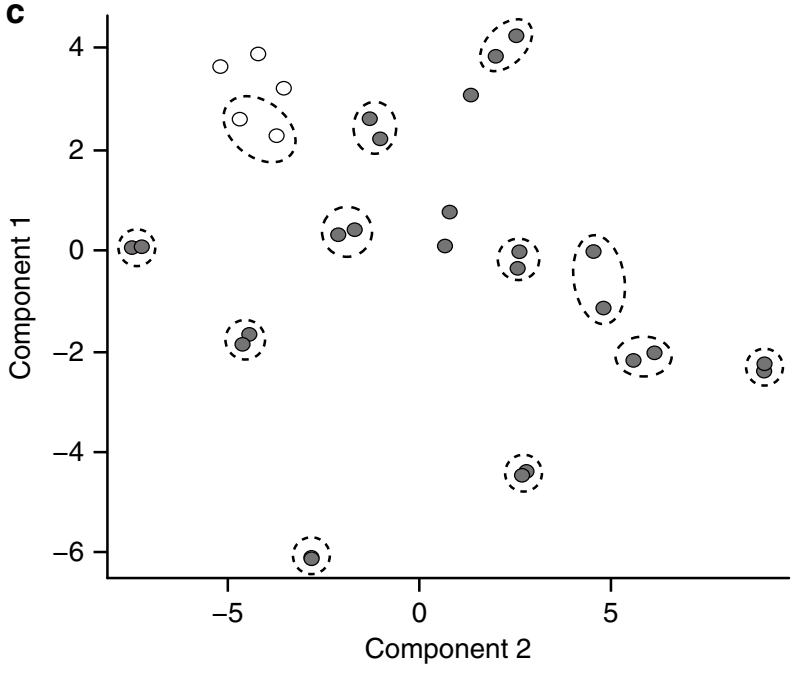

$\circ$ Ctrl $\circ \mathrm{Dmc1}^{-/-} \mathrm{Puma}^{-/-} \mathrm{Noxa}^{-/-}$

Fig. 4 Dmc1 $^{-1}-$ Puma $^{-1}-$ Noxa $^{-/}$- females generate embryos.

a Heatmap showing chromosome gains and losses in $\mathrm{Dmcl}^{-/-} \mathrm{Puma}^{-/-}$ $\mathrm{Noxa}^{-/-}$zygotes and their matched polar body, showing multiple reciprocal chromosomal aneuploidies. $\mathbf{b}$ Development of embryos from control ( $n=3$ females, $n=48$ zygotes, $N=2$ experimental repetitions) and

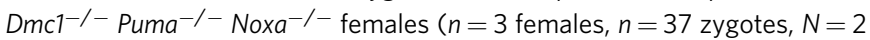
experimental repetitions). Dotted circles outline paternal and maternal pronuclei. Asterisks indicate second polar body. Time shows hours from zygote collection. Scale bar $100 \mu \mathrm{M}$. c $t$-SNE plot of single-cell RNA-seq analysis of control and $\mathrm{Dmcl}^{-/-}{ }^{-} \mathrm{Puma}^{-/}-\mathrm{Noxa}^{-/-}$blastomeres. Dotted circles represent pairs of blastomeres derived from the same embryo. Control (Ctrl) is $\mathrm{Dmcl}^{+/-} \mathrm{Puma}^{+/-} \mathrm{Noxa}^{+/-}$.
Puma $^{-1-} \mathrm{Noxa}^{-1-}$ mutant oocytes were associated with respective gains of material from the aneuploid chromosome in the corresponding zygote, indicating that the chromosome lost from the polar body had been retained by the oocyte. Multiple aneuploidies were present in all cases without a preference for particular chromosomes (Fig. 4a and Supplementary Fig. 4c). In contrast, all zygotes and polar bodies derived from control eggs were euploid ( $n=9$ zygotes; $n=9$ polar bodies).

Despite the presence of aneuploidy, zygotes derived from Dmcl $^{-1-}$ Puma $^{-1-}$ Noxa $^{-1-}$ mothers completed the first cleavage division (Fig. 4b). Live imaging revealed that the timing of cleavage relative to that of pronuclear fading was similar to that in controls (Supplementary Fig. 4d and Supplementary Movie 2). However, while embryos from control mothers continued to cleave, all those from Dmc1-I- $\mathrm{Puma}^{-1-} \mathrm{Noxa}^{-1-}$ mothers arrested at the two-cell stage (Fig. 4b). Furthermore, Dmc1-1Puma $^{-1-}$ Noxa $^{-1-}$ females produced no offspring $(n=4$ mating pairs). In mice, the first cleavage relies on maternal products, while development beyond the two-cell stage requires the activation of embryonic transcription ${ }^{39-42}$. We assayed transcription in blastomeres from late two-cell embryos using single-cell RNA-sequencing (RNA-seq). Unsupervised $t$-distributed stochastic neighbour embedding ( $t$-SNE) analysis showed that embryos derived from $\mathrm{Dmcl}^{-1-} \mathrm{Puma}^{-1-} \mathrm{Noxa}^{-1-}$ mothers did not cluster with those from control mothers (Fig. 4c). Furthermore, there was considerable transcriptional variability between individual Dmc1 $1^{-1-}$ Puma $^{-I-}$ Noxa $^{-/-}$-derived embryos, presumably resulting from their distinct aneuploid chromosome complements. Arrest at the two-cell stage in embryos derived from Dmc1 $1^{-1-} \mathrm{Puma}^{-1-} \mathrm{Noxa}^{-1-}$ mothers is therefore associated with chaotic gene expression.

Distinct apoptotic effectors operate in Spo11 nulls. Oocyte elimination occurs not only when programmed meiotic DSBs persist, but also when they are not formed. For example, Spo11 1 - females lack programmed DSBs, and this causes chromosome asynapsis and loss of oocytes around birth. Oocyte elimination in $S p o 11^{-/-}$females was attributed to a distinct, HORMAD1/2-dependent synapsis checkpoint ${ }^{15,16}$, and to meiotic silencing ${ }^{12}$, the inactivation of genes on asynapsed chromosomes. However, Spo11-1- oocytes exhibit markers of spontaneous DNA damage ${ }^{19}$, which could cause apoptosis via the DNA-damage checkpoint ${ }^{18}$. We therefore examined the effect of co-deleting Puma and Noxa, or deleting Bax alone, on oocyte loss in Spo11 1 - females. Interestingly, P21 primordial and total

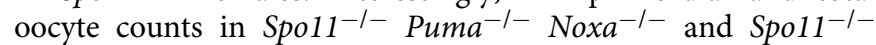
$\mathrm{Bax}^{-1-}$ females were similar to those in Spo11-1- females (Supplementary Fig. 5a, b). Thus, oocyte elimination in Spo11 $11^{-1-}$ females is independent of Puma, Noxa, and Bax. We conclude that the apoptotic effectors operating in Spo11-1- oocytes are genetically separable from those operating in $D m c 1^{-1-}$ and Msh5 ${ }^{-1-}$ oocytes.

\section{Discussion}

Here we identify a critical role for the BCL-2 apoptotic pathway in the mammalian oocyte DNA-damage checkpoint (Fig. 5). Integrating our work with others', we propose that residual meiotic DNA damage, repair of which is prevented during late prophase I by RNF212, activates $\mathrm{CHK} 2$, thereafter inducing PUMA/NOXA/BAX-dependent apoptosis. In Spo11-1- oocytes, chromosome asynapsis and/or spontaneous DNA damage signals via RNF212-stabilised HORMAD1/2 and CHK2, to distinct apoptotic effectors. The DNA-damage checkpoint no doubt comprises additional components (Fig. 5), because Puma/Noxa or Bax deletion, like Chk2 deletion ${ }^{18,43}$, does not restore oocyte 


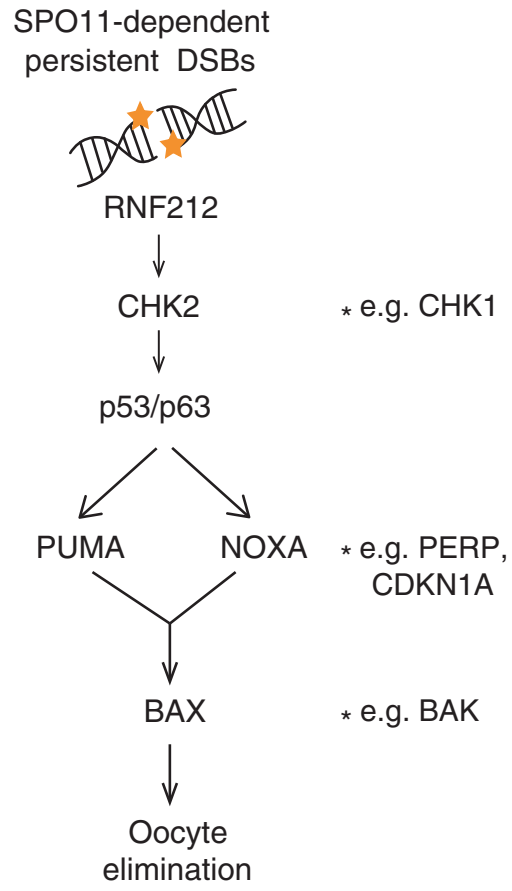

Fig. 5 Role of PUMA NOXA and BAX in the oocyte DNA-damage checkpoint. Asterisks represent additional checkpoint effectors that may contribute to the checkpoint.

numbers in DSB-repair mutants to wild type levels. Furthermore, we show that the oocyte responses to IR and meiotic DSBs are not exactly the same. Bax deletion fully rescues IR-but not persistent meiotic DSB-induced oocyte loss. Coexistence of multiple checkpoints may ensure a robust response to the wide variety of chromosomal defects that can arise during the protracted length of female prophase I. The findings raise the possibility that Bax inhibitors, in addition to Puma/Noxa inhibitors ${ }^{24}$, may be of utility in premature ovarian failure treatment and fertility preservation in women undergoing cancer therapy.

Our data support existing evidence 24,43 that in instances of compromised checkpoint activity, surviving oocytes exhibit some capacity to repair SPO11- or IR-induced DSBs. In $\mathrm{Dmcl}^{-1-}$ oocytes DSB focus counts diminish during prophase I exit, concomitant with disappearance of the axial element and HORMAD1, orthologues of which are negative regulators of intersister repair ${ }^{4-47}$. This point in oogenesis may mark a switch from a meiotic to a non-meiotic mechanism of DSB repair. Such repair may be beneficial in wild type oogenesis, where small numbers of lingering DSBs are insufficient to trigger oocyte elimination ${ }^{18}$, but could compromise embryonic viability. Deciphering whether DSB repair results in an elevated mutation frequency will be essential if Puma, Noxa, and Bax inhibitors are to be used in a clinical context.

\footnotetext{
Methods

Mice and animal irradiation. All animals were maintained with appropriate care according to the United Kingdom Animal Scientific Procedures Act 1986 and the ethics guidelines of the National Institute for Medical Research and Francis Crick Institute. Mice were housed in individually ventilated cages with free access to water and food. All studies were approved by local ethical review and UK Home Office. Genetically modified models are previously published: Puma ${ }^{48}, N_{0 x a}{ }^{48}$, and $S p o 11^{2}$ were maintained on C57BL/6 background; $D m c 1^{4}$ and $M s h 5^{6}$ were maintained on MF1 background, Bax mice ${ }^{49}$ were obtained from the Jackson Labs and are maintained on a mixed C57BL/6-129 background. Littermate controls were used where possible. For the irradiation experiment, P5 female pups were exposed to a single dose of ionising radiation $(0.45 \mathrm{~Gy})$ in a ${ }^{137}$ cesium irradiator.
}

Ovary section and surface spread immunofluorescence. Ovaries collected at P10 or P21 were fixed in 4\% paraformaldehyde (PFA) overnight at room temperature, washed in phosphate-buffered saline (PBS), embedded in paraffin, and serially sectioned at $6 \mu \mathrm{m}$. For ovary section immunofluorescence, slides were deparaffinised and re-hydrated using xylene and an ethanol series, followed by antigen retrieval for $30 \mathrm{~min}$ in $0.1 \mathrm{M}$ of sodium citrate buffer. Sections were blocked for $15 \mathrm{~min}$ in $5 \%$ bovine serum (PBS/Tween 20) and incubated at room temperature for $1 \mathrm{~h}$ with a germ cell marker (rabbit anti-MVH, 1:100, ab-13840 Abcam), followed by an incubation with secondary antibody (Alexa Fluor ${ }^{\circledR} 488$, 1:200) and DAPI for $1 \mathrm{~h}$ at room temperature. Slides were mounted with Vectashield (Thermo-Fisher). Images were obtained using Olympus VS120 Slide Scanner with a U-HGLGPS mercury lamp. An Olympus UPlanApo $20 \times / 1.35$ NA objective was used. Images were captured using $2 / 3^{\prime \prime}$ CCD camera and analysed using OlyVia Olympus software. For immunofluorescence, oocytes were treated with $0.05 \%$ Triton X-100 in water at room temperature for $10 \mathrm{~min}$, and fixed in $2 \%$ PFA, $0.02 \%$ sodium dodecyl sulfate in PBS at room temperature for $1 \mathrm{~h}$, washed in water, and air-dried. Slides were blocked in $0.15 \%$ bovine serum albumin, $0.1 \%$ Tween 20 in PBS at room temperature for $1 \mathrm{~h}$ and incubated with primary antibodies in a humidified chamber at $37^{\circ} \mathrm{C}$ overnight. Antibodies used were SYCP3 (ab-15092; Abcam), GCNA (ab-82527, Abcam), RPA32 (ab-10359; Abcam), and RAD51 (PC130 Calbiochem), used at 1:100, 1:50, 1:50, and 1:100, respectively ${ }^{50}$. Imaging was performed using an Olympus IX70 inverted microscope with a 100-W mercury arc lamp. An Olympus UPlanApo 100×/1.35 NA oil-immersion objective was used. Images were captured by using a DeltaVision RT computer-assisted Photometrics CoolSnap HQ CCD camera with an ICX285 Progressive scan CCD image sensor. Fiji software was used to process 8 - or 16 -bit $(512 \times 512$ or $1024 \times 1024$ pixels $)$ captured images.

Follicle quantification and fertility testing. For follicle quantification, every sixth section was examined for the presence of primordial, primary, secondary, and antral follicles ${ }^{51}$. One ovary per animal was used. Graphs and statistical analysis were performed with GraphPad Prism5. 6-8-week-old females were mated to proven fertile wild type males. Litter sizes were determined by counting pups on the day of birth.

In-vitro culture of mouse oocytes. Oocytes were collected from ovaries of 8-10 week-old mice and cultured at $37^{\circ} \mathrm{C}$ under mineral oil in homemade M2 medium or M16 medium (Sigma; MR-016), supplemented with $250 \mu \mathrm{M}$ dbcAMP (Sigma; D0627) to maintain prophase arrest. To trigger the resumption of meiosis, oocytes were released into dbcAMP-free medium.

Construct expression and live cell confocal microscopy. Capped mRNA was synthesised with T7 RNA polymerase (mMessage mMachine Kit Ambion), precipitated with isopropanol, and dissolved in $6 \mu \mathrm{l}$ of RNase-free water. The following constructs were used: pGEMHE-EGFP-MAP4 to label microtubules and pGEMHE- $H 2 B$-mRFP to visualise the chromosomes ${ }^{52}$. Quantitative microinjection was performed in a homemade chamber ${ }^{37}$. In brief, 10-15 oocytes were placed in a "microinjection slit" formed by separating two coverslips with a $100 \mu \mathrm{m}$ thick piece of double stick tape. The oocytes were injected with $10 \mathrm{pl}$ of mRNA. After injection of mRNA, the oocytes were immediately recovered from the chamber and the procedure was repeated for the remaining cells. Following the microinjection, the oocytes were incubated for $3 \mathrm{~h}$ at $37^{\circ} \mathrm{C}$ to express the fluorescently-labelled proteins. Thereafter, the oocytes were release into dbcAMP-free medium and imaged. Confocal images of live oocytes were acquired using Zeiss LSM800 microscope at $37.5^{\circ} \mathrm{C}$. Oocytes were imaged in M2 medium under oil using a $40 \times$ C-Apochromat 1.2 NA water-immersion objective. The samples were imaged at a temporal resolution of $10 \mathrm{~min}$ and optical slice thickness of $3 \mu \mathrm{m}$, covering $66 \mu \mathrm{m}$.

AiryScan immunofluorescence microscopy in fixed oocytes. Before fixation, kinetochore-bound microtubules were selectively depolymerised by exposing the oocytes to $4{ }^{\circ} \mathrm{C}$ for $14 \mathrm{~min}$. Following the cold-treatment, the dish was removed from ice and oocytes were permeabilised by a brief $10 \mathrm{~s}$ exposure to $0.25 \%$ Triton $\mathrm{X}-100$. Oocytes were then fixed for $30 \mathrm{~min}$ at $37^{\circ} \mathrm{C}$ in $100 \mathrm{mM}$ HEPES (pH 7; titrated with $\mathrm{KOH}$ ), $50 \mathrm{mM}$ EGTA ( $\mathrm{pH} 7$; titrated with $\mathrm{KOH}$ ), $2 \%$ formaldehyde (methanol-free), and $0.2 \%$ Triton X-100. Thereafter, oocytes were extracted overnight at $4{ }^{\circ} \mathrm{C}$ in PBS supplemented with $0.1 \%$ Triton X-100. All antibody incubations were performed in PBS, $3 \%$ bovine serum albumin, and $0.1 \%$ Triton X-100, either overnight at $4{ }^{\circ} \mathrm{C}$ (primary antibodies) or for $3 \mathrm{~h}$ at room temperature (secondary antibodies). Primary antibodies used were human ACA centromere CREST autoantibody (FZ90C-CS1058, Europa Bioproducts; 1:500) and rat anti- $\alpha$ tubulin (MCA78G, Serotec; 1:1000). As secondary antibodies, Alexa Fluor488 antihuman and Alexa Fluor647 anti-rat (Thermo Fisher; 1:400) were used. DNA was stained with $5 \mathrm{mg} / \mathrm{ml}$ Hoechst 33342 (Molecular Probes).

Fixed oocytes were imaged using the AiryScan module on Zeiss LSM800 microscope equipped with $40 \times \mathrm{C}$-Apochromat $1.2 \mathrm{NA}$ water-immersion objectives and processed post-acquisition using ZEN2. Images were acquired at a spatial resolution of $0.19-0.30 \mu \mathrm{m}$ optical sections, covering the entire spindle. 
Embryo collection and time lapse. 6-8-week-old timed mated females were used. Zygotes were collected from the oviduct at E0.5 and washed free of the cumulus cells through a brief treatment with $3 \mathrm{mg} / \mathrm{ml}$ hyaluronidase (Sigma; H4272), washed in FHM (MerckMillipore; MR-025-D) and cultured in drops of preequilibrated KSOM (MerckMillipore; MR-121-D) overlaid with mineral oil (Origio; ART-4008-5P). Embryos were incubated at $37^{\circ} \mathrm{C}$ and $5.5 \% \mathrm{CO}_{2}$ in an EmbryoScope+ time-lapse incubator (Vitrolife) for 1-2 days.

Dissociation of polar body and blastomeres. Embryos were washed in FHM and the zona pellucidae removed by a brief incubation in acidic tyrode's solution (Sigma; T1788), followed by washing in FHM containing 10\% serum supplement (Origio; ART-3001) and incubation in Accutase (ThermoFisher Scientific: A1110501) for 5-7 min. Polar bodies and blastomeres were washed in PBS and transferred to $0.5 \mathrm{ml}$ microfuge tubes containing $2 \mu \mathrm{l}$ of PBS, snap-frozen and stored at $-80^{\circ} \mathrm{C}$.

Low-coverage whole genome sequencing and aneuploidy analysis. Zygotes and polar bodies were subjected to lysis and whole genome amplification using SurePlex (Illumina). The amplified DNA was used to prepare libraries using the SQKLSK-108 kit (Oxford Nanopore Technologies) according to the manufacturer's instructions and sequenced on the MinION device (Oxford Nanopore Technologies), with the average number of mapped reads suitable for downstream analysis being approximately 200,000 per sample. Upon the completion of the sequencing run, reads which obtained a quality score of 7 or higher (determined as the threshold by the manufacturer), were demultiplexed using the Epi2Me software (Oxford Nanopore Technologies) and saved in fastq format. Adapters and barcodes were trimmed using the Porechop tools and the sequences were aligned to the Mus musculus genome (GRCm38_68) in Minimap2, using the default parameters ${ }^{53}$. BAM files were generated from SAM format, sorted and indexed in Samtools ${ }^{54}$. The reads were subsequently counted on a per chromosome basis in the Genome Analysis Tool Kit (Broad Institute) using the CountReads function ${ }^{55}$. The proportion of reads was then determined for each chromosome from the total number of aligned reads and compared to a reference set comprising data compiled from multiple karyotypically normal female and male samples. For all chromosomes in female samples, the resulting values were doubled prior to plotting them, generating the predicted copy number profiles, while for male samples only values from autosomes were transformed in this way, since $\mathrm{X}$ and $\mathrm{Y}$ chromosomes are each present in a single copy in euploid cells.

Preparation of single-cell libraries for RNA sequencing. Cells from cleaving embryos were individually collected in $5 \mathrm{ul}$ of PBSA, and used in the subsequent steps of cDNA synthesis and library construction. Single-cell cDNA was prepared using the SMART-Seq v4 Ultra Low Input RNA Kit (Cat. no. 634891, Clontech Laboratories), adhering to the manufacturer's protocol. Amplified double-stranded cDNA samples that had passed quality control checks were subsequently used to make libraries. A minimum of $1 \mathrm{ng}$ cDNA was used for the preparation of libraries, which was performed using the NexteraXT kit (Cat. no. FC -131-1096).

RNA sequencing, read processing. 32 Single-cell libraries were subjected to paired-end sequencing on the Illumina HiSEq 4000 analyser with 100 bp reads. Quality control was performed on FASTQ output files using the FastQC package ${ }^{56}$. Removal of adapters and low-quality bases from paired reads were performed using Trim Galore for each library, trimming of 11 base pairs from the $5^{\prime}$-end and 3 base pairs from the 3 '-end. Quality was checked once again using FastQC, which showed mean quality values across each base for reads from each library typically being above 37 , and the most frequently observed mean quality was 40 per sequence. Thus, trimmed paired-end reads were used in the subsequent analysis.

Analysis of RNA sequencing. Reads from each library were first aligned to the mouse genome (Grcm38) using HISAT2 v2.1.0 with the supplied index (genome_snp_tran $)^{57}$. Uniquely mapped reads (i.e., fragments that mapped once only to the reference genome) and with a MAPQ score $>40$ were retained for further analysis. These were then reverted to FASTQ format using Samtools (htslib 1.8) bam $2 \mathrm{fq}$ prior to input for further steps. Transcript abundances were determined using Salmon (v0.11.3) ${ }^{58}$ in transcripts per million (TPM). Mouse transcriptome annotation was obtained from Ensembl (ftp://ftp.ensembl.org/pub/release-94/fasta/ mus_musculus/cdna/Mus_musculus.GRCm38.cdna.all.fa.gz), which was used to create a quasi index.

Transcript abundances were then compiled and processed in R (v. 3.5.1). Briefly transcript abundances from samples were imported in the $\mathrm{R}$ environment using tximport ${ }^{59}$, and aggregated to gene-level abundances in TPM. Subsequent single cell analyses were performed using the Monocle package ${ }^{60}$.

Reporting summary. Further information on research design is available in the Nature Research Reporting Summary linked to this article.

\section{Data availability}

The Next-Generation Sequencing data is available on the ArrayExpress website under "EMTAB-8752" link. All data are available from the corresponding author upon reasonable request.

Received: 10 April 2019; Accepted: 27 April 2020;

Published online: 25 May 2020

\section{References}

1. Keeney, S., Giroux, C. N. \& Kleckner, N. Meiosis-specific DNA double-strand breaks are catalyzed by Spo11, a member of a widely conserved protein family. Cell 88, 375-384 (1997).

2. Baudat, F., Manova, K., Yuen, J. P., Jasin, M. \& Keeney, S. Chromosome synapsis defects and sexually dimorphic meiotic progression in mice lacking Spo11. Mol. Cell 6, 989-998 (2000).

3. Romanienko, P. J. \& Camerini-Otero, R. D. The mouse Spo11 gene is required for meiotic chromosome synapsis. Mol. Cell 6, 975-987 (2000).

4. Pittman, D. L. et al. Meiotic prophase arrest with failure of chromosome synapsis in mice deficient for Dmcl, a germline-specific RecA homolog. Mol. Cell 1, 697-705 (1998).

5. Yoshida, K. et al. The mouse RecA-like gene Dmc1 is required for homologous chromosome synapsis during meiosis. Mol. Cell 1, 707-718 (1998).

6. Edelmann, W. et al. Mammalian MutS homologue 5 is required for chromosome pairing in meiosis. Nat. Genet. 21, 123-127 (1999).

7. de Vries, S. S. et al. Mouse MutS-like protein Msh5 is required for proper chromosome synapsis in male and female meiosis. Genes Dev. 13, 523-531 (1999).

8. Cole, F. et al. Homeostatic control of recombination is implemented progressively in mouse meiosis. Nat. Cell Biol. 14, 424-430 (2012).

9. Nagaoka, S. I., Hassold, T. J. \& Hunt, P. A. Human aneuploidy: mechanisms and new insights into an age-old problem. Nat. Rev. Genet. 13, 493-504 (2012).

10. Morelli, M. A. \& Cohen, P. E. Not all germ cells are created equal: aspects of sexual dimorphism in mammalian meiosis. Reproduction 130, 761-781 (2005).

11. Handel, M. A. \& Schimenti, J. C. Genetics of mammalian meiosis: regulation, dynamics and impact on fertility. Nat. Rev. Genet. 11, 124-136 (2010).

12. Burgoyne, P. S., Mahadevaiah, S. K. \& Turner, J. M. The consequences of asynapsis for mammalian meiosis. Nat. Rev. Genet. 10, 207-216 (2009).

13. Cloutier, J. M. et al. Histone H2AFX links meiotic chromosome asynapsis to prophase I oocyte loss in mammals. PLoS Genet. 11, e1005462 (2015).

14. Di Giacomo, M. et al. Distinct DNA-damage-dependent and -independent responses drive the loss of oocytes in recombination-defective mouse mutants. Proc. Natl Acad. Sci. USA. 102, 737-742 (2005).

15. Wojtasz, L. et al. Meiotic DNA double-strand breaks and chromosome asynapsis in mice are monitored by distinct HORMAD2-independent and -dependent mechanisms. Genes Dev. 26, 958-973 (2012).

16. Daniel, $K$. et al. Meiotic homologue alignment and its quality surveillance are controlled by mouse HORMAD1. Nat. Cell Biol. 13, 599-610 (2011).

17. Qiao, H. et al. Impeding DNA break repair enables oocyte quality control Mol. Cell 72, 211-221 (2018).

18. Rinaldi, V. D., Bolcun-Filas, E., Kogo, H., Kurahashi, H. \& Schimenti, J. C. The DNA damage checkpoint eliminates mouse oocytes with chromosome synapsis failure. Mol. Cell 67, 1026-1036 (2017).

19. Carofiglio, F. et al. SPO11-independent DNA repair foci and their role in meiotic silencing. PLoS Genet. 9, e1003538 (2013).

20. Su, X., Chakravarti, D. \& Flores, E. R. p63 steps into the limelight: crucial roles in the suppression of tumorigenesis and metastasis. Nat. Rev. Cancer 13, 136-143 (2013).

21. Youle, R. J. \& Strasser, A. The BCL-2 protein family: opposing activities that mediate cell death. Nat. Rev. Mol. Cell Biol. 9, 47-59 (2008).

22. Willis, S. N. et al. Apoptosis initiated when BH3 ligands engage multiple Bcl-2 homologs, not Bax or Bak. Science 315, 856-859 (2007).

23. Youle, R. J. Cell biology. Cellular demolition and the rules of engagement. Science 315, 776-777 (2007).

24. Kerr, J. B. et al. DNA damage-induced primordial follicle oocyte apoptosis and loss of fertility require TAp63-mediated induction of Puma and Noxa. Mol. Cell 48, 343-352 (2012).

25. Perez, G. I. et al. Absence of the proapoptotic Bax protein extends fertility and alleviates age-related health complications in female mice. Proc. Natl Acad. Sci. USA. 104, 5229-5234 (2007).

26. Greenfeld, C. R., Pepling, M. E., Babus, J. K., Furth, P. A. \& Flaws, J. A. BAX regulates follicular endowment in mice. Reproduction 133, 865-876 (2007).

27. Ke, F. et al. Consequences of the combined loss of BOK and BAK or BOK and BAX. Cell Death Dis. 4, e650 (2013). 
28. Perez, G. I., Knudson, C. M., Leykin, L., Korsmeyer, S. J. \& Tilly, J. L. Apoptosis-associated signaling pathways are required for chemotherapymediated female germ cell destruction. Nat. Med. 3, 1228-1232 (1997).

29. Livera, G. et al. p63 null mutation protects mouse oocytes from radio-induced apoptosis. Reproduction 135, 3-12 (2008).

30. Suh, E. K. et al. p63 protects the female germ line during meiotic arrest. Nature 444, 624-628 (2006)

31. Sehorn, M. G., Sigurdsson, S., Bussen, W., Unger, V. M. \& Sung, P. Human meiotic recombinase Dmc1 promotes ATP-dependent homologous DNA strand exchange. Nature 429, 433-437 (2004).

32. Bishop, D. K., Park, D., Xu, L. \& Kleckner, N. DMC1: a meiosis-specific yeast homolog of $E$. coli recA required for recombination, synaptonemal complex formation, and cell cycle progression. Cell 69, 439-456 (1992).

33. Hollingsworth, N. M., Ponte, L. \& Halsey, C. MSH5, a novel MutS homolog, facilitates meiotic reciprocal recombination between homologs in Saccharomyces cerevisiae but not mismatch repair. Genes Dev. 9, 1728-1739 (1995).

34. Gray, S. \& Cohen, P. E. Control of meiotic crossovers: from double-strand break formation to designation. Annu. Rev. Genet. 50, 175-210 (2016).

35. Su, Y. Q. et al. Oocyte-dependent activation of mitogen-activated protein kinase (ERK1/2) in cumulus cells is required for the maturation of the mouse oocyte-cumulus cell complex. Dev. Biol. 263, 126-138 (2003).

36. Pfender, S. et al. Live imaging RNAi screen reveals genes essential for meiosis in mammalian oocytes. Nature 524, 239-242 (2015).

37. Jaffe, L. A., Norris, R. P., Freudzon, M., Ratzan, W. J. \& Mehlmann, L. M. Microinjection of follicle-enclosed mouse oocytes. Methods Mol. Biol. 518, 157-173 (2009).

38. Herbert, M., Kalleas, D., Cooney, D., Lamb, M. \& Lister, L. Meiosis and maternal aging: insights from aneuploid oocytes and trisomy births. Cold Spring Harb. Perspect. Biol. 7, a017970 (2015).

39. Latham, K. E., Garrels, J. I., Chang, C. \& Solter, D. Quantitative analysis of protein synthesis in mouse embryos. I. Extensive reprogramming at the oneand two-cell stages. Development 112, 921-932 (1991).

40. Bensaude, O., Babinet, C., Morange, M. \& Jacob, F. Heat shock proteins, first major products of zygotic gene activity in mouse embryo. Nature 305, 331-333 (1983).

41. Flach, G., Johnson, M. H., Braude, P. R., Taylor, R. A. \& Bolton, V. N. The transition from maternal to embryonic control in the 2-cell mouse embryo. EMBO J. 1, 681-686 (1982).

42. Conover, J. C., Temeles, G. L., Zimmermann, J. W., Burke, B. \& Schultz, R. M. Stage-specific expression of a family of proteins that are major products of zygotic gene activation in the mouse embryo. Dev. Biol. 144, 392-404 (1991).

43. Bolcun-Filas, E., Rinaldi, V. D., White, M. E. \& Schimenti, J. C. Reversal of female infertility by Chk2 ablation reveals the oocyte DNA damage checkpoint pathway. Science 343, 533-536 (2014).

44. Wojtasz, L. et al. Mouse HORMAD1 and HORMAD2, two conserved meiotic chromosomal proteins, are depleted from synapsed chromosome axes with the help of TRIP13 AAA-ATPase. PLoS Genet. 5, e1000702 (2009).

45. Carballo, J. A., Johnson, A. L., Sedgwick, S. G. \& Cha, R. S. Phosphorylation of the axial element protein Hopl by Mec1/Tell ensures meiotic interhomolog recombination. Cell 132, 758-770 (2008).

46. Niu, H. et al. Partner choice during meiosis is regulated by Hop1-promoted dimerization of Mek1. Mol. Biol. Cell 16, 5804-5818 (2005).

47. Hodges, C. A., LeMaire-Adkins, R. \& Hunt, P. A. Coordinating the segregation of sister chromatids during the first meiotic division: evidence for sexual dimorphism. J. Cell Sci. 114, 2417-2426 (2001).

48. Villunger, A. et al. p53- and drug-induced apoptotic responses mediated by BH3-only proteins puma and noxa. Science 302, 1036-1038 (2003).

49. Knudson, C. M., Tung, K. S., Tourtellotte, W. G., Brown, G. A. \& Korsmeyer, S. J. Bax-deficient mice with lymphoid hyperplasia and male germ cell death. Science 270, 96-99 (1995).

50. Turner, J. M. et al. Silencing of unsynapsed meiotic chromosomes in the mouse. Nat. Genet. 37, 41-47 (2005).

51. Myers, M., Britt, K. L., Wreford, N. G., Ebling, F. J. \& Kerr, J. B. Methods for quantifying follicular numbers within the mouse ovary. Reproduction 127, 569-580 (2004).

52. Schuh, M. \& Ellenberg, J. Self-organization of MTOCs replaces centrosome function during acentrosomal spindle assembly in live mouse oocytes. Cell 130, 484-498 (2007).

53. Li, H. Minimap2: pairwise alignment for nucleotide sequences. Bioinformatics 34, 3094-3100 (2018).

54. Li, H. et al. The Sequence Alignment/Map format and SAMtools. Bioinformatics 25, 2078-2079 (2009).

55. McKenna, A. et al. The Genome Analysis Toolkit: a MapReduce framework for analyzing next-generation DNA sequencing data. Genome Res. 20, 1297-1303 (2010).
56. Andrews, S. FastQC: A Quality Control Tool for High Throughput Sequence Data (2010)

57. Kim, D., Langmead, B. \& Salzberg, S. L. HISAT: a fast spliced aligner with low memory requirements. Nat. Methods 12, 357-360 (2015).

58. Patro, R., Duggal, G., Love, M. I., Irizarry, R. A. \& Kingsford, C. Salmon provides fast and bias-aware quantification of transcript expression. Nat. Methods 14, 417-419 (2017).

59. Soneson, C., Love, M. I. \& Robinson, M. D. Differential analyses for RNA-seq transcript-level estimates improve gene-level inferences. F1000Res 4, 1521 (2015).

60. Trapnell, C. et al. The dynamics and regulators of cell fate decisions are revealed by pseudotemporal ordering of single cells. Nat. Biotechnol. 32, 381-386 (2014)

\section{Acknowledgements}

This work was supported by European Research Council (CoG 647971 to J.M.A.T.) and the Francis Crick Institute, which receives its core funding from Cancer Research UK (FC001193 to J.M.A.T. and FC001120 to K.K.N.), UK Medical Research Council (FC001193 and FC001120), and Wellcome Trust (FC001193 and FC001120). A.P.Z. and M.S. received financial support from the Max Planck Society, the European Research Council (CoG 337415), the Deutsche Forschungsgemeinschaft (Leibniz Prize to M.S.), and the Lister Institute for Preventive Medicine. A.P.Z. is further supported by a Rosetrees Trust PhD Fellowship. D.W. was supported by the NIHR Oxford Biomedical Research Centre. N.K. was supported by the University of Oxford Clarendon Fund and Brasenose College Joint Scholarship. The authors thank the Francis Crick Institute Biological Research, Advanced Sequencing, Light Microscopy and Experimental Histopathology facilities for their expertise; Andreas Strasser and Andreas Wyttenbach for the Puma $^{-I-}$ and Noxa ${ }^{-1-}$ mice; Eva Hoffmann, Jenny Gruhn, and Keith Jones for advice on oocyte preparation and analysis; Attila Toth for discussion of data; and Nitzan Gonen, Emily Frost, Scott Keeney, Francesca Cole, and members of the J.M.A.T and K.K.N. laboratories for comments on the manuscript.

\section{Author contributions}

E.E., J.M.A.T., M.S., K.K.N., and D.W. conceived the project. E.E., S.M., and V.M. performed immunofluorescence. E.E. and O.O. generated mouse models and assessed their fertility. E.E. and A.P.Z. performed counting, in vitro culture, and confocal microscopy of live and fixed oocytes. E.E. and A.M. performed collection, timelapse, and single-cell harvesting of embryos. E.E. created Fig. 5. N.K. performed low-coverage whole genome sequencing and aneuploidy analysis. M.N.S. performed single-cell RNA-seq analysis. E.E. and J.M.A.T. wrote the manuscript.

\section{Competing interests}

The authors declare no competing interests.

\section{Additional information}

Supplementary information is available for this paper at https://doi.org/10.1038/s41467020-16441-z.

Correspondence and requests for materials should be addressed to J.M.A.T

Peer review information Nature Communications thanks the anonymous reviewer(s) for their contribution to the peer review of this work.

Reprints and permission information is available at http://www.nature.com/reprints

Publisher's note Springer Nature remains neutral with regard to jurisdictional claims in published maps and institutional affiliations.

Open Access This article is licensed under a Creative Commons Attribution 4.0 International License, which permits use, sharing, adaptation, distribution and reproduction in any medium or format, as long as you give appropriate credit to the original author(s) and the source, provide a link to the Creative Commons license, and indicate if changes were made. The images or other third party material in this article are included in the article's Creative Commons license, unless indicated otherwise in a credit line to the material. If material is not included in the article's Creative Commons license and your intended use is not permitted by statutory regulation or exceeds the permitted use, you will need to obtain permission directly from the copyright holder. To view a copy of this license, visit http://creativecommons.org/ licenses/by/4.0/.

(c) The Author(s) 2020 